\begin{tabular}{c} 
Volume and Issues Obtainable at Center for Sustainability Research and Consultancy \\
Journal of Business and Social Review in Emerging Economies \\
ISSN: 2519-089X (E): 2519-0326 \\
Volume 6: No. 1, March 2020 \\
CSRᄃ \\
Journal homepage: www.publishing.globalcsrc.org/jbsee \\
\hline
\end{tabular}

\title{
Investigating Critical Resemblances of Islamic Banking with Conventional: Binary Matrices as Solution Methodology
}

\author{
${ }^{1}$ Abdul Aziz Khan Niazi, ${ }^{2}$ Tehmina Fiaz Qazi, ${ }^{3}$ Abdul Basit \\ ${ }^{1}$ Institute of Business and Management, University of Engineering and Technology, Lahore, Pakistan \\ azizniazi@uet.edu.pk \\ ${ }^{2}$ Institute of Business and Management, University of Engineering and Technology, Lahore, Pakistan \\ tehmina.qazi@gmail.com \\ ${ }^{3}$ Lahore Institute of Science and Technology, Lahore, Pakistan. abasit_shahbaz@yahoo.com
}

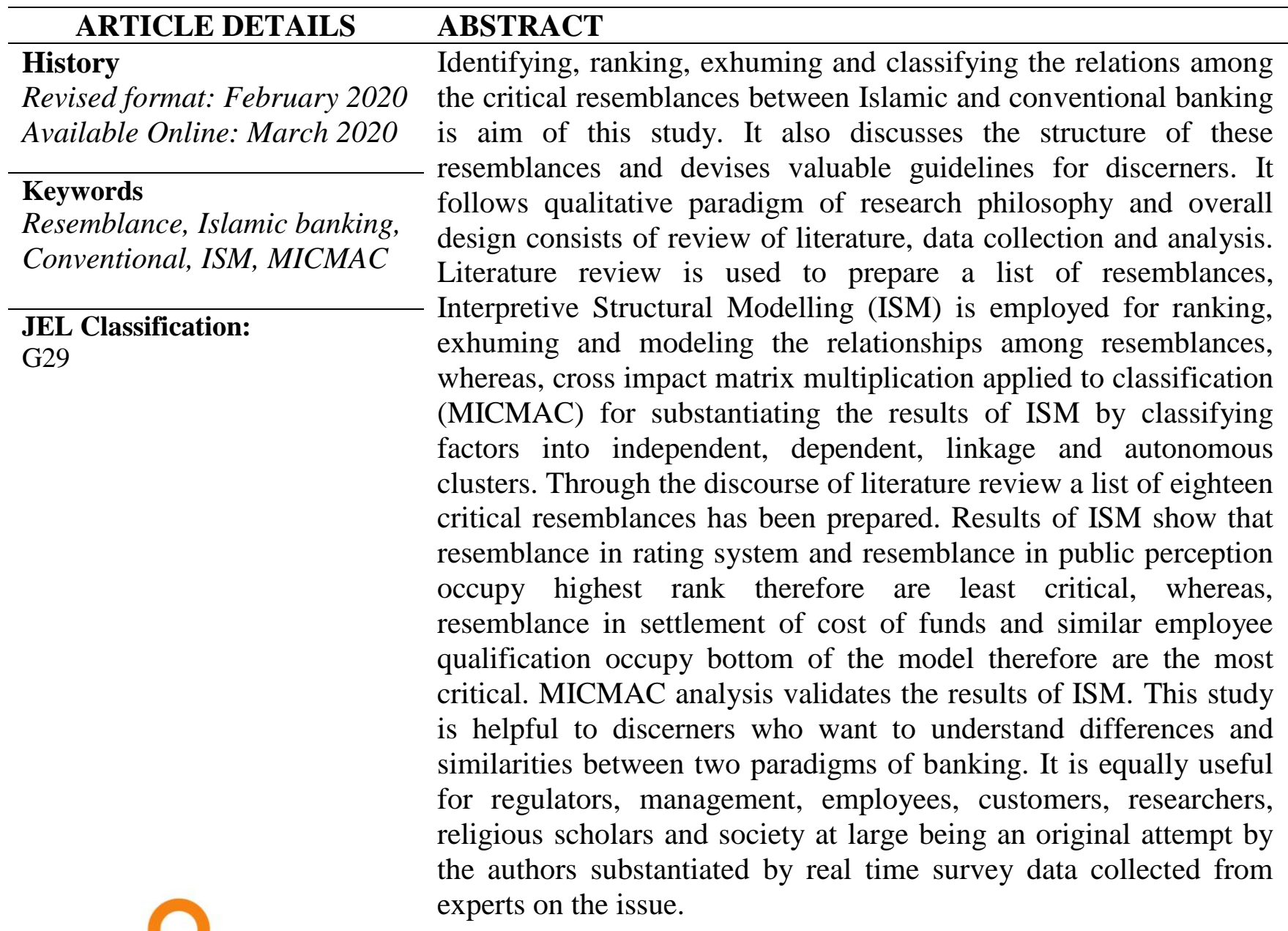

OPEN ACCESS

(C) 2020 The authors, under a Creative Commons Attribution-

NonCommercial 4.0

Corresponding author's email address: azizniazi@uet.edu.pk

Recommended citation: Niazi, A.A.K., Qazi, T.F., Basit. A. (2020). Investigating Critical Resemblances of Islamic Banking with Conventional: Binary Matrices as Solution Methodology. Journal of Business and Social Review in Emerging Economies, 6(1), 349-364

DOI: $10.26710 /$ jafee.v6i1.1045 


\section{Introduction}

Much effort of theorists and researchers has been devoted to refine differences and resemblances between Islamic and conventional banking. It is a hot topic in the area of banking particularly when both co-exist in many countries. Conventional banks dominate banking business whereas advocates of Islamic banks expect gradual paradigm shift in clientele. There are many reasons for believing them similar or different that may be religious or non-religious, but to date discerners are confused and are unable to separate the two on immaculate basis. Theoretical clarifications, given to date, do not seem to be plausible. It is indispensable to study similarities and dissimilarities of Islamic and conventional banking in order to corroborate the contemporary findings. Ahmad and Haron (2002) asserted that the products of Islamic banking are not whole heartedly accepted because they cannot be clearly distinguished from that of conventional. Khan and Mirakhor (1994) concluded that there is no such true profit and loss sharing system. Beck et al. (2011) bolstered that Islamic banks' business model is not too different from that of conventional. It also represented that it is difficult for the discerners to clearly distinguish between the two paradigms of banking. Zeitun and Benjelloun (2012) argued that Islamic banks offer many products having high degree of resemblance with that of conventional banks. Samad (2004) also concluded that there is no material difference as such between financial performance of two categories of banks. Ahmad et al. (2010) emphasized that Muslims still patronize conventional banks for their faster and efficient services.

In fact, there is resemblance between Islamic and conventional banks on many counts that have not been scientifically studied. There is lack of researches investigating the phenomenon in holistic, comprehensive and integrative way to make position rather clearer. It is important to investigate the issue of resemblance of Islamic banking with conventional because stakeholders are confused on the issue. Hence the objectives of this study are: i) to identify and rank the resemblances between Islamic and conventional banking, ii) to exhume interactions among the factors, iii) to enlighten upon that how structural model is helpful to distinguish between two paradigms of banking, iv) to deliberate theoretical and practical implications by way of classification of factors and v) to propose framework for future research. To achieve these objectives, the study considered different methodological choices. In order to ascertain critical resemblances, it considered ten different methods viz: literature review (Dhochak \& Sharma, 2016; Li et al., 2019; Song et al., 2017), expert opinion (Dhochak \& Sharma, 2016), case study (Li et al., 2019; Valmohammadi \& Dashti, 2016), Delphi method (Bhosale \& Kant, 2016; Zhang \& Wei, 2010), exploratory factor analysis (Li \& Yang, 2014), meta-analysis (Lohaus \& Habermann, 2019), presuming by authors (Lohaus \& Habermann, 2019), idea engineering and brainstorming session (Kumar et al., 2013), interview content analysis (Xiao, 2018) and anecdotal evidences (Azevedo et al., 2013). In this study the method of literature review coupled with expert opinion has been considered as appropriate. It is the most commonly used methods for these types of studies. For structuring of the issue grounded theory, thematic content analysis, structural equation modelling and interpretive structural modelling have been considered. Interpretive structural modelling has been found best to commensurate to objectives of the study (Warfield, 1973 \& 1974; Sushil, 2017). In order to confirm the results of structural modelling another technique namely MICMAC has been employed to substantiate results of ISM. Rest of the paper is arranged as literature review, solution methodology, results, discussion and conclusion.

\section{Literature Review}

Before embarking on analysis, the study accounted for reasonable amount of literature apropos to the issue. Out of thorough literature survey some germane studies are being reported here to set out the outset of the study. Lee and Isa (2017) bolstered that Islamic and conventional banks have similarities with slight differences. Salman and Nawaz (2018) carried a study in order to identify difference among Islamic and conventional banking concerning efficiency, liquidity and profitability. It signifies that customer trust in Islamic banking is more influenced by return on asset than in conventional banking. Amin et al. (2013) asserted that customer satisfaction with regards to trust, customer loyalty and image in Islamic and conventional banks are significantly interrelated; while differences exist in terms of Muslim and non- 
Muslim customers. Karim et al. (2014) argued that high requirements of capital influences deposit and lending actions of Islamic and conventional banks in similar manner. Rizvi and Arshad (2014) established that derivatives and proximate alternatives in Islamic finance are, in fact, not in compliance with the values and framework of Shariah rather they resemble with that of conventional, however, a more recent literature suggests some tools complying with Shariah for mitigating risks of Islamic finance. Rhanoui and Belkhoutout (2018) focused on how Islamic and conventional banks perceive operational risks objectifying to determine the extent to which operational risk is similar and different and found that most of the operational risks are similar.

Barnett and Iqbal (2013) highlighted similarities between Islamic finance and Socially Responsible Investing (SRI). During the last two decades, Islamic finance has witnessed a much impressive growth rate, yet the Islamic fixed income market is lagging behind which also has resemblance with that of conventional banking. Al-Mamun et al. (2014) concluded that there are plenty of differences among interest-based conventional banking system and interest-free (Islamic) banking system. Islamic banking system adopts moral, social and ethical ways of creating wealth and gives second priority to economic benefits; whereas conventional believes in creating wealth through pure economic i.e. almost ignoring ethical, moral and social means. Hanif (2014) delineated that Islamic banks have gained customers' trust and obtained deposits on the basis of profit and loss sharing yet the options of financing and investment available for Islamic banks are still similar to and minimal than conventional banks. Ibrahim and Ismail (2015) identified differences between both banking areas in terms of foundation law, economic functions and business organization. Ariff and Lewis (2014) proclaimed that there are few similarities between Shariah and common law but major differences exist among them by which it can be concluded that there are more differences among both laws than the similarities.

Islamic banking is deflecting from its primary path of considering moral and social aspects by embracing operational practices of conventional banking. There is minor difference among them concerning financial risks and deposit rates but there is no significant difference in monthly average rates of lending (Ahmed et al., 2014; Azmat et al., 2017; Jawadi et al., 2016). Saidu (2014) found that there is a difference between principles for Islamic banking and its actual working. Khan (2010) argued that regulatory reforms are required instead of introducing a separate law for Islamic banking.

\begin{tabular}{cll}
\hline Sr. & \multicolumn{1}{c}{ Resemblances } & \multicolumn{1}{c}{ Author(s) } \\
\hline $\mathbf{1}$ & Resemblance in Settlement of Cost of Funds & (Lee \& Isa, 2017) \\
$\mathbf{2}$ & Resemblance in Determination of Rates of Profits & (Haron \& Ahmad, 2000) \\
$\mathbf{3}$ & Resemblance in Sanction Procedures & (Lee \& Isa, 2017) \\
$\mathbf{4}$ & Resemblance in Repayment Annuities & (Lee \& Isa, 2017) \\
$\mathbf{5}$ & Resemblance in Documentation Processes & (Lee \& Isa, 2017) \\
$\mathbf{6}$ & Resemblance in Working Styles & (Ahmad \& Hassan, 2006) \\
$\mathbf{7}$ & Resemblance in Interbank Clearings & (Ismath Bacha, 2008) \\
$\mathbf{8}$ & Resemblance in Borrowing Patterns & (Lee \& Isa, 2017) \\
$\mathbf{9}$ & Resemblance in Accounting Practices & (Karim, 2001) \\
$\mathbf{1 0}$ & Same Banking Laws/Rules/Regulations & (Ariff \& Lewis, 2014) \\
$\mathbf{1 1}$ & Resemblance in Rating System & (Hadriche, 2015) \\
$\mathbf{1 2}$ & Resemblance in Public Perception & (Hanif et al., 2012) \\
$\mathbf{1 3}$ & Resemblance in Client Assessment Procedure & (Hanif et al., 2012) \\
$\mathbf{1 4}$ & Resemblance in Banker's Behavior & (Zainol et al., 2009) \\
$\mathbf{1 5}$ & Resemblance in Recovery Procedures & (Ahmed et al., 2006) \\
$\mathbf{1 6}$ & Similar Employee Qualification & (Bashir et at., 2011) \\
$\mathbf{1 7}$ & Resemblance in HRM Practices & (Bowra et al., 2012) \\
$\mathbf{1 8}$ & Similar Marketing Tactics & (Saeed \& Baig, 2013) \\
\hline
\end{tabular}

Table 1: List of Resemblances in Islamic and Conventional Banking 
The practices of modern Islamic banking and financial institutions are violating the real Islamic principles and thus have failed in their claim to be a thorough alternative of conventional banking. Zarrouk et al. (2016) revealed that Islamic banking positively influences profitability in Middle East and North Africa (MENA) region yet many profitability antecedents of Islamic and conventional banks are same. It is also argued that changing the perspective of non-Muslims is major obstacle in evolving Islamic banking in its true letter and spirit. Non-Muslims view Islamic banking merely as a religious system that should only be used by Muslims (Badruddin, 2015; Omercic, 2016). However, some conventional banks are converting from conventional to Islamic that due to continuous demand for Islamic products and compliance with Shariah principles (Asif et al., 2017). With the support of aforementioned representation of literature total eighteen resemblances have been identified (Table 1) which were ratified by experts. Same have been used for data collection and analyses.

\section{Solution Methodology}

This study follows qualitative approach. Overall design consists of selective literature review, survey for data collection and data analysis. It is a cross sectional experimental study using primary type of data elicited directly from stakeholders viz: Shariah scholars, Islamic bankers, conventional bankers, customers of Islamic banking, customers of conventional banking, regulators and researchers. Literature discourse is used to set the outset of the study and factor identification, ISM has been employed for imposing structure on the elements (resemblances here in this study) of the issue and MICMAC analysis for classification based on driving and dependence power. ISM and MICMAC analyses are mathematical techniques that used theory of binary matrices in combination with elementary concepts of set theory. The methodologies follow principle of Boolean Algebra for basic mathematical operations.

Interpretive Structural Modeling (ISM): ISM is a well-defined methodology for construction of model using multiplication properties of binary matrices. It is visible modeling approach using reachability and transitive inferences through matrix transformation. ISM applies when there is no priory theoretical framework and the situation is conundrum like that of we have in hand. ISM uses the data collected from experts on the issue.

Panel of Experts in ISM: Panel of experts means the persons conversant with issue. In this case panel have been recruited from within the stakeholders aforementioned. This type of sampling is useful when data are not-existing, expensive, limited, unreliable and confusing (Ranjbar et al., 2012). In this case opinion of experts is considered to be valid. The expert opinions outperform as compare to statistical archival data (Clayton, 1997). Experts have been recruited on the criteria of their theoretical knowledge, practical experience (minimum 10 years), expert knowledge and organization in which they are working admitting the fact that quality is more important than quantity of experts. The panel is comprised of sixteen experts (Clayton, 1997; Khan \& Khan, 2013) having expertise on different aspects of the issue. We have developed rapport with experts as piloting. We approached more than thirty experts, only twenty-one agreed but actually participated sixteen. Experts first have been briefed on the issue in their office setting. Two to three discussion sessions were held with experts to persuade them to participate in the study and those who were agreeable were approached face-to-face one-on-one basis ( $\mathrm{Li} \& \mathrm{Yang}$, 2014). Many visits were paid to experts for completing survey. A matrix type questionnaire suitable to ISM was used to elicit the data using factor $i$ leads to factor $j$. It took more than two months to approach experts and collect data. Standard symbols (i.e. V,A,O,X) have been used to record data. Data was consolidated using the principle "minority gives way to majority" (Abdullah \& Siraj 2014; Cai \& Xia 2018; Dhochak \& Sharma, 2016; Li et al., 2019; Sushil 2012). The panel of experts was engaged at three different levels i.e. first for the judgement as to whether the factors are reasonable and representative of phenomenon, second for determination of contextual relationships and third for reviewing the model qua reality. We proceeded to ISM stepwise (Attri et al., 2013; Thakkar et al., 2008; Warfield, 1973) as follows: 
Step 1 Identification of factors

Step 2 Development of Structural Self-Interaction Matrix (SSIM)

Step 3 Establishing the contextual relationships among elements

Step 4 Development of the final reachability matrix

Step 5 Partitioning the reachability

Step 6 Development of conical matrix

Step 7 Development of digraph

Step 1 Identification of factors: Already performed in section of literature review.

Step 2 Development of Structural Self-Interaction Matrix (SSIM): The data collected on matrix type questionnaire has been converted into SSIM using principle of majority. SSIM contains data in $i$-j part in form of VAXO that follows the rules as given below:

Establishing the contextual relationship between factors

\begin{tabular}{|c|c|c|c|c|}
\hline $\begin{array}{l}\text { Rules for questionnaire/SSIM: } \\
\text { Rules for reachability matrix: }\end{array}$ & $V: i \rightarrow j$ & $A: i \longleftarrow j$ & $X: i \longleftrightarrow j$ & $O: i \triangleleft \mid j$ \\
\hline for $i-j$ entry & 1 & 0 & 1 & 0 \\
\hline for $j-i$ entry & 0 & 1 & 1 & 0 \\
\hline
\end{tabular}

Figure 1: Rules of construction of SSIM and Initial Reachability Matrix

Table 2: Structural Self-Interaction Matrix

\begin{tabular}{|c|c|c|c|c|c|c|c|c|c|c|c|c|c|c|c|c|c|c|c|}
\hline & Resemblances & 1 & 2 & 3 & 4 & 5 & 6 & 7 & 8 & 9 & 10 & 11 & 12 & 13 & 14 & 15 & 16 & 17 & 18 \\
\hline 1 & $\begin{array}{l}\text { Resemblance in Settlement of Cost of } \\
\text { Funds }\end{array}$ & & V & $\mathrm{O}$ & $\mathrm{O}$ & $\mathrm{O}$ & $\mathrm{O}$ & A & V & $\mathrm{O}$ & A & $\mathrm{O}$ & V & $\mathrm{O}$ & $\mathrm{O}$ & $\mathrm{O}$ & $\mathrm{O}$ & $\mathrm{O}$ & $\mathrm{O}$ \\
\hline 2 & $\begin{array}{l}\text { Resemblance in Determination of Rates of } \\
\text { Profits }\end{array}$ & & & $\mathrm{O}$ & $\mathrm{V}$ & $\mathrm{O}$ & $\mathrm{O}$ & A & $\mathrm{O}$ & $\mathrm{X}$ & A & $\mathrm{O}$ & V & $\mathrm{O}$ & $\mathrm{O}$ & $\mathrm{O}$ & $\mathrm{O}$ & $\mathrm{V}$ & $\mathrm{O}$ \\
\hline 3 & Resemblance in Sanction Procedures & & & & $\mathrm{X}$ & $\mathrm{X}$ & $\mathrm{X}$ & $\mathrm{O}$ & $\mathrm{O}$ & V & A & $\mathrm{O}$ & V & $\mathrm{X}$ & $\mathrm{O}$ & $\mathrm{V}$ & A & $\mathrm{V}$ & A \\
\hline 4 & Resemblance in Repayment Annuities & & & & & V & $\mathrm{O}$ & $\mathrm{O}$ & V & A & $\mathrm{O}$ & $\mathrm{V}$ & $\mathrm{O}$ & $\mathrm{O}$ & $\mathrm{V}$ & $\mathrm{O}$ & $\mathrm{O}$ & $\mathrm{O}$ & $\mathrm{X}$ \\
\hline 7 & Resemblance in Interbank Clearings & & & & & & & & A & V & A & V & V & $\mathrm{O}$ & $\mathrm{O}$ & $\mathrm{O}$ & $\mathrm{O}$ & $\mathrm{O}$ & $\mathrm{O}$ \\
\hline 8 & Resemblance in Borrowing Patterns & & & & & & & & & $\mathrm{V}$ & $\mathrm{V}$ & $\mathrm{V}$ & A & $\mathrm{V}$ & $\mathrm{O}$ & $\mathrm{V}$ & $\mathrm{O}$ & $\mathrm{O}$ & $\mathrm{O}$ \\
\hline 9 & Resemblance in Accounting Practices & & & & & & & & & & A & $\mathrm{V}$ & V & $\mathrm{O}$ & $\mathrm{O}$ & $\mathrm{O}$ & A & $\mathrm{V}$ & $\mathrm{O}$ \\
\hline 10 & Same Banking Laws/Rules/Regulations & & & & & & & & & & & V & V & $\mathrm{V}$ & $\mathrm{V}$ & $\mathrm{V}$ & $\mathrm{O}$ & $\mathrm{V}$ & $\mathrm{V}$ \\
\hline 14 & Resemblance in Banker's Behavior & & & & & & & & & & & & & & & V & A & A & $\mathrm{O}$ \\
\hline 15 & $\begin{array}{l}\text { Resemblance } \\
\text { in Recovery Procedures }\end{array}$ & & & & & & & & & & & & & & & & $\mathrm{O}$ & $\mathrm{O}$ & $\mathrm{O}$ \\
\hline 16 & Similar Employee Qualification & & & & & & & & & & & & & & & & & $\mathrm{V}$ & $\mathrm{V}$ \\
\hline 17 & Resemblance in HRM Practices & & & & & & & & & & & & & & & & & & $\mathrm{O}$ \\
\hline 18 & Similar Marketing Tactics & & & & & & & & & & & & & & & & & & \\
\hline
\end{tabular}

Step 3 Establishing the contextual relationship among elements: For establishing the contextual relationships initial reachability matrix has been prepared using the rules as enumerated in Figure 1. 
Table 3: Initial Reachability Matrix

\begin{tabular}{llllllllllllllllllll}
\hline & $\mathbf{1}$ & $\mathbf{2}$ & $\mathbf{3}$ & $\mathbf{4}$ & $\mathbf{5}$ & $\mathbf{6}$ & $\mathbf{7}$ & $\mathbf{8}$ & $\mathbf{9}$ & $\mathbf{1 0}$ & $\mathbf{1 1}$ & $\mathbf{1 2}$ & $\mathbf{1 3}$ & $\mathbf{1 4}$ & $\mathbf{1 5}$ & $\mathbf{1 6}$ & $\mathbf{1 7}$ & $\mathbf{1 8}$ & Driving \\
\hline $\mathbf{1}$ & 1 & 1 & 0 & 0 & 0 & 0 & 0 & 1 & 0 & 0 & 0 & 1 & 0 & 0 & 0 & 0 & 0 & 0 & 4 \\
$\mathbf{2}$ & 0 & 1 & 0 & 1 & 0 & 0 & 0 & 0 & 1 & 0 & 0 & 1 & 0 & 0 & 0 & 0 & 1 & 0 & 5 \\
$\mathbf{3}$ & 0 & 0 & 1 & 1 & 1 & 1 & 0 & 0 & 1 & 0 & 0 & 1 & 1 & 0 & 1 & 0 & 1 & 0 & 9 \\
$\mathbf{4}$ & 0 & 0 & 1 & 1 & 1 & 0 & 0 & 1 & 0 & 0 & 1 & 0 & 0 & 1 & 0 & 0 & 0 & 1 & 7 \\
$\mathbf{5}$ & 0 & 0 & 1 & 0 & 1 & 1 & 0 & 1 & 1 & 0 & 0 & 1 & 0 & 1 & 1 & 0 & 0 & 0 & 8 \\
$\mathbf{6}$ & 0 & 0 & 1 & 0 & 1 & 1 & 0 & 0 & 0 & 0 & 0 & 1 & 1 & 0 & 1 & 0 & 1 & 1 & 8 \\
$\mathbf{7}$ & 1 & 1 & 0 & 0 & 0 & 0 & 1 & 0 & 1 & 0 & 1 & 1 & 0 & 0 & 0 & 0 & 0 & 0 & 6 \\
$\mathbf{8}$ & 0 & 0 & 0 & 0 & 1 & 0 & 1 & 1 & 1 & 1 & 1 & 0 & 1 & 0 & 1 & 0 & 0 & 0 & 8 \\
$\mathbf{9}$ & 0 & 1 & 0 & 1 & 0 & 1 & 0 & 0 & 1 & 0 & 1 & 1 & 0 & 0 & 0 & 0 & 1 & 0 & 7 \\
$\mathbf{1 0}$ & 1 & 1 & 1 & 0 & 1 & 1 & 1 & 0 & 1 & 1 & 1 & 1 & 1 & 1 & 1 & 0 & 1 & 1 & 15 \\
$\mathbf{1 1}$ & 0 & 0 & 0 & 0 & 0 & 0 & 0 & 0 & 0 & 0 & 1 & 1 & 0 & 0 & 0 & 0 & 0 & 0 & 2 \\
$\mathbf{1 2}$ & 0 & 0 & 0 & 0 & 0 & 0 & 0 & 1 & 0 & 0 & 0 & 1 & 0 & 0 & 0 & 0 & 0 & 0 & 2 \\
$\mathbf{1 3}$ & 0 & 0 & 1 & 0 & 1 & 0 & 0 & 0 & 0 & 0 & 1 & 1 & 1 & 1 & 1 & 0 & 0 & 0 & 7 \\
$\mathbf{1 4}$ & 0 & 0 & 0 & 0 & 0 & 1 & 0 & 0 & 0 & 0 & 0 & 1 & 1 & 1 & 1 & 0 & 0 & 0 & 5 \\
$\mathbf{1 5}$ & 0 & 0 & 0 & 0 & 0 & 0 & 0 & 0 & 0 & 0 & 0 & 1 & 1 & 0 & 1 & 0 & 0 & 0 & 3 \\
$\mathbf{1 6}$ & 0 & 0 & 1 & 0 & 0 & 1 & 0 & 0 & 1 & 0 & 0 & 1 & 0 & 1 & 0 & 1 & 1 & 1 & 8 \\
$\mathbf{1 7}$ & 0 & 0 & 0 & 0 & 0 & 1 & 0 & 0 & 0 & 0 & 0 & 1 & 0 & 1 & 0 & 0 & 1 & 0 & 4 \\
$\mathbf{1 8}$ & 0 & 0 & 0 & 1 & 0 & 0 & 0 & 0 & 0 & 0 & 0 & 1 & 0 & 0 & 0 & 0 & 0 & 1 & 3
\end{tabular}

$\begin{array}{lllllllllllllllllll}\text { Dependence } & 3 & 5 & 7 & 5 & 7 & 8 & 3 & 5 & 8 & 2 & 7 & 16 & 7 & 7 & 8 & 1 & 7 & 5\end{array}$

Step 4 Development of the final reachability matrix: Initial reachability matrix has been converted into final reachability by removing transitivity as per norms of ISM (Table 4).

Table 4: Final Reachability Matrix

\begin{tabular}{|c|c|c|c|c|c|c|c|c|c|c|c|c|c|c|c|c|c|c|c|}
\hline & 1 & 2 & 3 & 4 & 5 & 6 & 7 & 8 & 9 & 10 & 11 & 12 & 13 & 14 & 15 & 16 & 17 & 18 & Driving \\
\hline 1 & 1 & 1 & 0 & $1 *$ & $1 *$ & 0 & $1 *$ & 1 & $1 *$ & $1 *$ & $1 *$ & 1 & $1 *$ & 0 & $1 *$ & 0 & $1 *$ & 0 & 13 \\
\hline 2 & 0 & 1 & $1 *$ & 1 & $1 *$ & $1 *$ & 0 & $1 *$ & 1 & 0 & $1 *$ & 1 & 0 & $1 *$ & 0 & 0 & 1 & $1 *$ & 12 \\
\hline 3 & 0 & $1 *$ & 1 & 1 & 1 & 1 & 0 & $1 *$ & 1 & 0 & $1 *$ & 1 & 1 & $1 *$ & 1 & 0 & 1 & $1 *$ & 14 \\
\hline 4 & 0 & 0 & 1 & 1 & 1 & $1 *$ & $1 *$ & 1 & $1 *$ & $1 *$ & 1 & $1 *$ & $1 *$ & 1 & $1 *$ & 0 & $1^{*}$ & 1 & 15 \\
\hline 5 & 0 & $1 *$ & 1 & $1 *$ & 1 & 1 & $1 *$ & 1 & 1 & $1 *$ & $1 *$ & 1 & $1 *$ & 1 & 1 & 0 & $1 *$ & $1 *$ & 16 \\
\hline 6 & 0 & 0 & 1 & $1 *$ & 1 & 1 & $1 *$ & $1 *$ & $1 *$ & 0 & $1 *$ & 1 & 1 & $1 *$ & 1 & 0 & 1 & 1 & 14 \\
\hline 7 & 1 & 1 & 0 & $1 *$ & 0 & $1 *$ & 1 & $1 *$ & 1 & 0 & 1 & 1 & 0 & 0 & 0 & 0 & $1^{*}$ & 0 & 10 \\
\hline 8 & $1 *$ & $1 *$ & $1 *$ & $1 *$ & 1 & $1 *$ & 1 & 1 & 1 & 1 & 1 & $1 *$ & 1 & $1 *$ & 1 & 0 & $1^{*}$ & 0 & 16 \\
\hline 9 & 0 & 1 & $1 *$ & 1 & $1 *$ & 1 & 0 & 0 & 1 & $1 *$ & 1 & 1 & $1 *$ & $1 *$ & $1 *$ & 0 & 1 & $1 *$ & 14 \\
\hline 10 & 1 & 1 & 1 & $1 *$ & 1 & 1 & 1 & $1^{*}$ & 1 & 1 & 1 & 1 & 1 & 1 & 1 & 0 & 1 & 1 & 17 \\
\hline 11 & 0 & 0 & 0 & 0 & 0 & 0 & 0 & $1^{*}$ & 0 & 0 & 1 & 1 & 0 & 0 & 0 & 0 & 0 & 0 & 3 \\
\hline 12 & 0 & 0 & 0 & 0 & $1 *$ & 0 & $1 *$ & 1 & 0 & $1 *$ & $1 *$ & 1 & $1^{*}$ & 0 & $1 *$ & 0 & 0 & 0 & 8 \\
\hline 13 & 0 & 0 & 1 & $1^{*}$ & 1 & $1^{*}$ & 0 & $1^{*}$ & $1 *$ & 0 & 1 & 1 & 1 & 1 & 1 & 0 & $1^{*}$ & 0 & 12 \\
\hline 14 & 0 & 0 & $1 *$ & 0 & $1^{*}$ & 1 & 0 & $1^{*}$ & 0 & 0 & $1^{*}$ & 1 & 1 & 1 & 1 & 0 & 0 & 0 & 9 \\
\hline 15 & 0 & 0 & $1 *$ & 0 & $1 *$ & 0 & 0 & $1 *$ & 0 & 0 & $1 *$ & 1 & 1 & $1^{*}$ & 1 & 0 & 0 & 0 & 8 \\
\hline 16 & 0 & 0 & 1 & $1 *$ & $1^{*}$ & 1 & 0 & $1 *$ & 1 & 0 & 0 & 1 & 0 & 1 & $1 *$ & 1 & 1 & 1 & 12 \\
\hline
\end{tabular}




\begin{tabular}{cccccccccccccccccccc}
\hline $\mathbf{1 7}$ & 0 & 0 & $1^{*}$ & 0 & $1^{*}$ & 1 & 0 & $1^{*}$ & 0 & 0 & 0 & 1 & $1^{*}$ & 1 & $1^{*}$ & 0 & 1 & $1^{*}$ & 10 \\
$\mathbf{1 8}$ & 0 & 0 & $1^{*}$ & 1 & $1^{*}$ & 0 & 0 & $1^{*}$ & 0 & 0 & $1^{*}$ & 1 & 0 & $1^{*}$ & 0 & 0 & 0 & 1 & 8 \\
Dependence & 4 & 8 & 14 & 13 & 16 & 13 & 8 & 17 & 12 & 7 & 16 & 18 & 13 & 14 & 14 & 1 & 13 & 10 & \\
\hline
\end{tabular}

Step 5 Partitioning the reachability matrix: Binary matrix given as Table 4 has been partitioned (Table 5 - 13) using the elementary concepts of set theory according to Warfeild (1973).

Table 5: Iteration I

\begin{tabular}{cccc}
\hline $\mathbf{S r}$ & Reachability Set & Antecedent Set & Intersection Set \\
\hline $\mathbf{1}$ & $1,2,4,5,7,8,9,10,11,12,13,15,17$ & $1,7,8,10$ & $1,7,8,10$ \\
$\mathbf{2}$ & $2,3,4,5,6,8,9,11,12,14,17,18$ & $1,2,3,5,7,8,9,10$ & $2,3,5,8,9$ \\
$\mathbf{3}$ & $2,3,4,5,6,8,9,11,12,13,14,15,17,18$ & $2,3,4,5,6,8,9,10,13,14,15,16,17,18$ & $2,3,4,5,6,8,9,13,14,15,17,18$ \\
$\mathbf{4}$ & $3,4,5,6,7,8,9,10,11,12,13,14,15,17,18$ & $1,2,3,4,5,6,7,8,9,10,13,16,18$ & $3,4,5,6,7,8,9,10,13,18$ \\
$\mathbf{5}$ & $2,3,4,5,6,7,8,9,10,11,12,13,14,15,17,18$ & $1,2,3,4,5,6,8,9,10,12,13,14,15,16,17,18$ & $2,3,4,5,6,8,9,10,12,13,14,15,17,18$ \\
$\mathbf{6}$ & $3,4,5,6,7,8,9,11,12,13,14,15,17,18$ & $2,3,4,5,6,7,8,9,10,13,14,16,17$ & $3,4,5,6,7,8,9,13,14,17$ \\
$\mathbf{7}$ & $1,2,4,6,7,8,9,11,12,17$ & $1,4,5,6,7,10,12$ & $1,4,6,7,12$ \\
$\mathbf{8}$ & $1,2,3,4,5,6,7,8,9,10,11,12,13,14,15,17$ & $1,2,3,4,5,6,7,8,10,11,12,13,14,15,16,17,18$ & $1,2,3,4,5,6,7,8,10,11,12,13,14,15$ \\
$\mathbf{9}$ & $2,3,4,5,6,9,10,11,12,13,14,15,17,18$ & $1,2,3,4,5,6,7,8,9,10,13,16$ & $2,3,4,5,6,9,10,13$ \\
$\mathbf{1 0}$ & $1,2,3,4,5,6,7,8,9,10,11,12,13,14,15,17,18$ & $1,4,5,9,10,12$ & $1,4,5,9,10,12$ \\
$\mathbf{1 1}$ & $8,11,12$ & $1,2,3,4,5,6,7,8,9,10,11,12,13,14,15,18$ & $8,11,12$ \\
$\mathbf{1 2}$ & $5,7,8,10,11,12,13,15$ & $1,2,3,4,5,6,7,8,9,10,11,12,13,14,15,16,17,18$ & $5,7,8,10,11,12,13,15$ \\
$\mathbf{1 3}$ & $3,4,5,6,8,9,11,12,13,14,15,17$ & $1,3,4,5,6,8,9,10,12,13,14,15,17$ & $3,4,5,6,8,9,12,13,14,15,17$ \\
$\mathbf{1 4}$ & $3,5,6,8,11,12,13,14,15$ & $2,3,4,5,6,8,9,10,13,14,15,16,17,18$ & $3,5,6,8,13,14,15$ \\
$\mathbf{1 5}$ & $3,5,8,11,12,13,14,15$ & $1,3,4,5,6,8,9,10,12,13,14,15,16,17$ & $3,5,8,12,13,14,15$ \\
$\mathbf{1 6}$ & $3,4,5,6,8,9,12,14,15,16,17,18$ & 16 & 16 \\
$\mathbf{1 7}$ & $3,5,6,8,12,13,14,15,17,18$, & $1,2,3,4,5,6,7,8,9,10,13,16,17$ & $3,5,6,8,13,17$ \\
$\mathbf{1 8}$ & $3,4,5,8,11,12,14,18$ & $2,3,4,5,6,9,10,16,18$ & $3,4,5,18$ \\
\hline
\end{tabular}

Table 6: Iteration II

\begin{tabular}{|c|c|c|c|c|}
\hline $\begin{array}{l}\mathrm{Sr} \\
.\end{array}$ & Reachability Set & Antecedent Set & Intersection Set & $\begin{array}{l}\text { Leve } \\
1\end{array}$ \\
\hline 1 & $1,2,4,5,7,8,9,10,13,15,17$ & $1,7,8,10$ & $1,7,8,10$ & \\
\hline 2 & $2,3,4,5,6,8,9,14,17,18$ & $1,2,3,5,7,8,9,10$ & $2,3,5,8,9$ & \\
\hline 3 & $2,3,4,5,6,8,9,13,14,15,17,18$ & $2,3,4,5,6,8,9,10,13,14,15,16,17,18$ & $2,3,4,5,6,8,9,13,14,15,17,18$ & \\
\hline 4 & $3,4,5,6,7,8,9,10,13,14,15,17,18$ & $1,2,3,4,5,6,7,8,9,10,13,16,18$ & $3,4,5,6,7,8,9,10,13,18$ & \\
\hline 5 & $2,3,4,5,6,7,8,9,10,13,14,15,17,18$ & $\begin{array}{l}1,2,3,4,5,6,8,9,10 \\
13,14,15,16,17,18\end{array}$ & $\begin{array}{c}2,3,4,5,6,8,9,10,13,14,15,17, \\
18\end{array}$ & \\
\hline 6 & $3,4,5,6,7,8,9,13,14,15,17,18$ & $2,3,4,5,6,7,8,9,10,13,14,16,17$ & $3,4,5,6,7,8,9,13,14,17$ & \\
\hline 7 & $1,2,4,6,7,8,9,17$ & $1,4,5,6,7,10$ & $1,4,6,7$ & \\
\hline 8 & $1,2,3,4,5,6,7,8,9,10,13,14,15,17$ & $\begin{array}{c}1,2,3,4,5,6,7,8,10,13,14,15,16,17 \\
18\end{array}$ & $1,2,3,4,5,6,7,8,10,13,14,15$ & \\
\hline 9 & $2,3,4,5,6,9,10,13,14,15,17,18$ & $1,2,3,4,5,6,7,8,9,10,13,16$ & $2,3,4,5,6,9,10,13$ & \\
\hline 10 & $\begin{array}{c}1,2,3,4,5,6,7,8,9,10,13,14,15,17 \\
18\end{array}$ & $1,4,5,9,10$ & $1,4,5,9,10$ & \\
\hline 13 & $3,4,5,6,8,9,13,14,15,17$ & $1,3,4,5,6,8,9,10,13,14,15,17$ & $3,4,5,6,8,9,13,14,15,17$ & II \\
\hline 14 & $3,5,6,8,13,14,15$ & $2,3,4,5,6,8,9,10,13,14,15,16,17,18$ & $3,5,6,8,13,14,15$ & II \\
\hline 15 & $3,5,8,13,14,15$ & $1,3,4,5,6,8,9,10,13,14,15,16,17$ & $3,5,8,13,14,15$ & II \\
\hline 16 & $3,4,5,6,8,9,14,15,16,17,18$ & 16 & 16 & \\
\hline 17 & $3,5,6,8,13,14,15,17,18$ & $1,2,3,4,5,6,7,8,9,10,13,16,17$ & $3,5,6,8,13,17$ & \\
\hline 18 & $3,4,5,8,14,18$ & $2,3,4,5,6,9,10,16,18$ & $3,4,5,18$ & \\
\hline
\end{tabular}


Table 7: Iteration III

\begin{tabular}{ccccc}
\hline Sr. & Reachability Set & Antecedent Set & Intersection Set & Level \\
\hline $\mathbf{1}$ & $1,2,4,5,7,8,9,10,17$ & $1,7,8,10$ & $1,7,8,10$ & \\
$\mathbf{2}$ & $2,3,4,5,6,8,9,17,18$ & $1,2,3,5,7,8,9,10$ & $2,3,5,8,9$ & \\
$\mathbf{3}$ & $2,3,4,5,6,8,9,17,18$ & $2,3,4,5,6,8,9,10,16,17,18$ & $2,3,4,5,6,8,9,17,18$ & III \\
$\mathbf{4}$ & $3,4,5,6,7,8,9,10,18$ & $1,2,3,4,5,6,7,8,9,10,16,18$ & $3,4,5,6,7,8,9,10,18$ & III \\
$\mathbf{5}$ & $2,3,4,5,6,7,8,9,10,17,18$ & $1,2,3,4,5,6,8,9,10,16,17,18$ & $2,3,4,5,6,8,9,10,17,18$ & \\
$\mathbf{6}$ & $3,4,5,6,7,8,9,17,18$ & $2,3,4,5,6,7,8,9,10,16,17$ & $3,4,5,6,7,8,9,17$ & \\
$\mathbf{7}$ & $1,2,4,6,7,8,9,17$ & $1,4,5,6,7,10$, & $1,4,6,7$ & \\
$\mathbf{8}$ & $1,2,3,4,5,6,7,8,9,10,17$ & $1,2,3,4,5,6,7,8,10,16,17,18$ & $1,2,3,4,5,6,7,8,10$ & \\
$\mathbf{9}$ & $2,3,4,5,6,9,10,17,18$ & $1,2,3,4,5,6,7,8,9,10,16$ & $2,3,4,5,6,9,10$ & \\
$\mathbf{1 0}$ & $1,2,3,4,5,6,7,8,9,10,17,18$ & $1,4,5,9,10$ & $1,4,5,9,10$ & \\
$\mathbf{1 6}$ & $3,4,5,6,8,9,16,17,18$ & 16 & 16 & \\
$\mathbf{1 7}$ & $3,5,6,8,17,18$, & $1,2,3,4,5,6,7,8,9,10,16,17$ & $3,5,6,8,17$ & \\
$\mathbf{1 8}$ & $3,4,5,8,18$ & $2,3,4,5,6,9,10,16,18$ & $3,4,5,18$ & III \\
\hline
\end{tabular}

Table 8: Iteration IV

\begin{tabular}{ccccc}
\hline Sr. & Reachability Set & Antecedent Set & Intersection Set & Level \\
\hline $\mathbf{1}$ & $1,2,5,7,8,9,10,17$ & $1,7,8,10$ & $1,7,8,10$ & \\
$\mathbf{2}$ & $2,5,6,8,9,17$ & $1,2,5,7,8,9,10$ & $2,5,8,9$ & \\
$\mathbf{5}$ & $2,5,6,7,8,9,10,17$ & $1,2,5,6,8,9,10,16,17$ & $2,5,6,8,9,10,17$ & \\
$\mathbf{6}$ & $5,6,7,8,9,17$ & $2,5,6,7,8,9,10,16,17$ & $5,6,7,8,9,17$ & IV \\
$\mathbf{7}$ & $1,2,6,7,8,9,17$ & $1,5,6,7,10$, & $1,6,7$ & \\
$\mathbf{8}$ & $1,2,5,6,7,8,9,10,17$ & $1,2,5,6,7,8,10,16,17$ & $1,2,5,6,7,8,10$ & \\
$\mathbf{9}$ & $2,5,6,9,10,17$ & $1,2,5,6,7,8,9,10,16$ & $2,5,6,9,10$ & \\
$\mathbf{1 0}$ & $1,2,5,6,7,8,9,10,17$ & $1,5,9,10$ & $1,5,9,10$ & \\
$\mathbf{1 6}$ & $5,6,8,9,16,17$ & 16 & 16 & \\
$\mathbf{1 7}$ & $5,6,8,17$ & $1,2,3,4,5,6,7,8,9,10,16,17$ & $3,5,6,8,17$ & $\mathrm{IV}$ \\
\hline
\end{tabular}

Table 9: Iteration V

\begin{tabular}{ccccc}
\hline Sr. & Reachability Set & Antecedent Set & Intersection Set & Level \\
\hline $\mathbf{1}$ & $1,2,5,7,8,9,10$ & $1,7,8,10$ & $1,7,8,10$ & \\
$\mathbf{2}$ & $2,5,8,9$ & $1,2,5,7,8,9,10$ & $2,5,8,9$ & $\mathrm{~V}$ \\
$\mathbf{5}$ & $2,5,7,8,9,10$ & $1,2,5,8,9,10,16,17$ & $2,5,8,9,10$ & \\
$\mathbf{7}$ & $1,2,7,8,9$ & $1,5,6,7,10$, & 1,7 & \\
$\mathbf{8}$ & $1,2,5,7,8,9,10$, & $1,2,5,7,8,10,16$ & $1,2,5,7,8,10$ & \\
$\mathbf{9}$ & $2,5,9,10$ & $1,2,5,7,8,9,10,16$ & $2,5,9,10$ & $\mathrm{~V}$ \\
$\mathbf{1 0}$ & $1,2,5,7,8,9,10$ & $1,5,9,10$ & $1,5,9,10$ & \\
$\mathbf{1 6}$ & $5,8,9,16$ & 16 & 16 & \\
\hline
\end{tabular}

Table 10: Iteration VI

\begin{tabular}{ccccc}
\hline Sr. & Reachability Set & Antecedent Set & Intersection Set & Level \\
\hline $\mathbf{1}$ & $1,5,7,8,10$ & $1,7,8,10$ & $1,7,8,10$ & \\
$\mathbf{5}$ & $5,7,8,10$ & $1,5,8,10,16$ & $5,8,10$ & \\
$\mathbf{7}$ & $1,7,8$ & $1,5,6,7,10$ & 1,7 & \\
$\mathbf{8}$ & $1,5,7,8,10$ & $1,5,7,8,10,16$ & $1,5,7,8,10$ & VI \\
$\mathbf{1 0}$ & $1,5,7,8,10$ & $1,5,10$ & $1,5,10$ & \\
$\mathbf{1 6}$ & $5,8,16$ & 16 & 16 & \\
\hline
\end{tabular}

Table 11: Iteration VII

\begin{tabular}{ccccc}
\hline Sr. & Reachability Set & Antecedent Set & Intersection Set & Level \\
\hline $\mathbf{1}$ & $1,5,7,10$ & $1,7,10$ & $1,7,10$ & \\
$\mathbf{5}$ & $5,7,10$ & $1,5,10,16$ & 5,10 & \\
$\mathbf{7}$ & 1,7 & $1,5,6,7,10$ & 1,7 & VII \\
$\mathbf{1 0}$ & $1,5,7,10$ & $1,5,10$ & $1,5,10$ & \\
\hline
\end{tabular}




\begin{tabular}{ccccc}
\hline $\mathbf{1 6}$ & 5,16 & 16 & 16 \\
\hline \multicolumn{5}{c}{ Table 12: Iteration VIII } \\
\hline Sr. & Reachability Set & Antecedent Set & Intersection Set & Level \\
\hline $\mathbf{1}$ & $1,5,10$ & 1,10 & 1,10 & \\
$\mathbf{5}$ & 5,10 & $1,10,16$ & 5,10 & VIII \\
$\mathbf{1 0}$ & $1,5,10$ & $1,5,10$ & $1,5,10$ & VIII \\
$\mathbf{1 6}$ & 5,16 & 16 & 16 & \\
\hline
\end{tabular}

Table 13: Iteration IX

\begin{tabular}{ccccc}
\hline Sr. & Reachability Set & Antecedent Set & Intersection Set & Level \\
\hline $\mathbf{1}$ & 1 & 1 & 1 & IX \\
$\mathbf{1 6}$ & 16 & 16 & 16 & IX \\
\hline
\end{tabular}

Step 6 Development of conical matrix: Conical matrix has been constructed by swapping column $j_{n}$ with $j_{n}$ and swapping column $i_{n}$ with $i_{n}$ according to Warfield (1973).

Table 14: Conical Matrix

\begin{tabular}{|c|c|c|c|c|c|c|c|c|c|c|c|c|c|c|c|c|c|c|c|}
\hline & 11 & 12 & 13 & 14 & 15 & 3 & 4 & 18 & 6 & 17 & 2 & 9 & 8 & 7 & 5 & 10 & 1 & 16 & Driving \\
\hline 11 & 1 & 1 & 0 & 0 & 0 & 0 & 0 & 0 & 0 & 0 & 0 & 0 & $1^{*}$ & 0 & 0 & 0 & 0 & 0 & 3 \\
\hline 12 & $1^{*}$ & 1 & $1^{*}$ & 0 & $1^{*}$ & 0 & 0 & 0 & 0 & 0 & 0 & 0 & 1 & $1^{*}$ & $1^{*}$ & $1^{*}$ & 0 & 0 & 8 \\
\hline 13 & 1 & 1 & 1 & 1 & 1 & 1 & $1^{*}$ & 0 & $1^{*}$ & $1^{*}$ & 0 & $1^{*}$ & $1^{*}$ & 0 & 1 & 0 & 0 & 0 & 12 \\
\hline 14 & $1^{*}$ & 1 & 1 & 1 & 1 & $1^{*}$ & 0 & 0 & 1 & 0 & 0 & 0 & $1^{*}$ & 0 & $1^{*}$ & 0 & 0 & 0 & 9 \\
\hline 15 & $1^{*}$ & 1 & 1 & 1* & 1 & $1 *$ & 0 & 0 & 0 & 0 & 0 & 0 & $1^{*}$ & 0 & $1^{*}$ & 0 & 0 & 0 & 8 \\
\hline 3 & $1^{*}$ & 1 & 1 & $1^{*}$ & 1 & 1 & 1 & $1^{*}$ & 1 & 1 & $1 *$ & 1 & $1^{*}$ & 0 & 1 & 0 & 0 & 0 & 14 \\
\hline 4 & 1 & $1^{*}$ & $1^{*}$ & 1 & $1^{*}$ & 1 & 1 & 1 & $1^{*}$ & $1^{*}$ & 0 & $1^{*}$ & 1 & $1^{*}$ & 1 & $1^{*}$ & 0 & 0 & 15 \\
\hline 18 & $1^{*}$ & 1 & 0 & $1^{*}$ & 0 & $1^{*}$ & 1 & 1 & 0 & 0 & 0 & 0 & $1^{*}$ & 0 & $1^{*}$ & 0 & 0 & 0 & 8 \\
\hline 6 & $1^{*}$ & 1 & 1 & $1^{*}$ & 1 & 1 & $1^{*}$ & 1 & 1 & 1 & 0 & $1^{*}$ & $1^{*}$ & $1^{*}$ & 1 & 0 & 0 & 0 & 14 \\
\hline 17 & 0 & 1 & $1^{*}$ & 1 & $1 *$ & $1^{*}$ & 0 & $1^{*}$ & 1 & 1 & 0 & 0 & $1^{*}$ & 0 & $1^{*}$ & 0 & 0 & 0 & 10 \\
\hline 2 & $1^{*}$ & 1 & 0 & $1^{*}$ & 0 & $1^{*}$ & 1 & $1^{*}$ & $1^{*}$ & 1 & 1 & 1 & $1^{*}$ & 0 & $1^{*}$ & 0 & 0 & 0 & 12 \\
\hline 9 & 1 & 1 & $1^{*}$ & $1^{*}$ & $1^{*}$ & $1^{*}$ & 1 & $1^{*}$ & 1 & 1 & 1 & 1 & 0 & 0 & $1^{*}$ & $1^{*}$ & 0 & 0 & 14 \\
\hline 8 & 1 & $1^{*}$ & 1 & $1^{*}$ & 1 & $1^{*}$ & $1^{*}$ & 0 & $1^{*}$ & $1^{*}$ & $1 *$ & 1 & 1 & 1 & 1 & 1 & $1^{*}$ & 0 & 16 \\
\hline 7 & 1 & 1 & 0 & 0 & 0 & 0 & $1^{*}$ & 0 & $1^{*}$ & $1^{*}$ & 1 & 1 & $1^{*}$ & 1 & 0 & 0 & 1 & 0 & 10 \\
\hline 5 & $1^{*}$ & 1 & $1^{*}$ & 1 & 1 & 1 & $1^{*}$ & $1^{*}$ & 1 & $1 *$ & $1^{*}$ & 1 & 1 & $1 *$ & 1 & $1^{*}$ & 0 & 0 & 16 \\
\hline 10 & 1 & 1 & 1 & 1 & 1 & 1 & $1^{*}$ & 1 & 1 & 1 & 1 & 1 & $1^{*}$ & 1 & 1 & 1 & 1 & 0 & 17 \\
\hline 1 & $1 *$ & 1 & $1^{*}$ & 0 & $1^{*}$ & 0 & $1^{*}$ & 0 & 0 & $1^{*}$ & 1 & $1^{*}$ & 1 & $1^{*}$ & $1 *$ & $1^{*}$ & 1 & 0 & 13 \\
\hline 16 & 0 & 1 & 0 & 1 & $1^{*}$ & 1 & $1^{*}$ & 1 & 1 & 1 & 0 & 1 & $1^{*}$ & 0 & $1^{*}$ & 0 & 0 & 1 & 12 \\
\hline Dependence & 16 & 18 & 13 & 14 & 14 & 14 & 13 & 10 & 13 & 13 & 8 & 12 & 17 & 8 & 16 & 7 & 4 & 1 & \\
\hline
\end{tabular}

Step 7 Development of digraph: The level determined through iterations aforementioned and arrangement of the level by way of swapping rows and columns of final reachability matrix and a model appeared on diagonal. It has been presented in form of a directed graph Figure 2. 


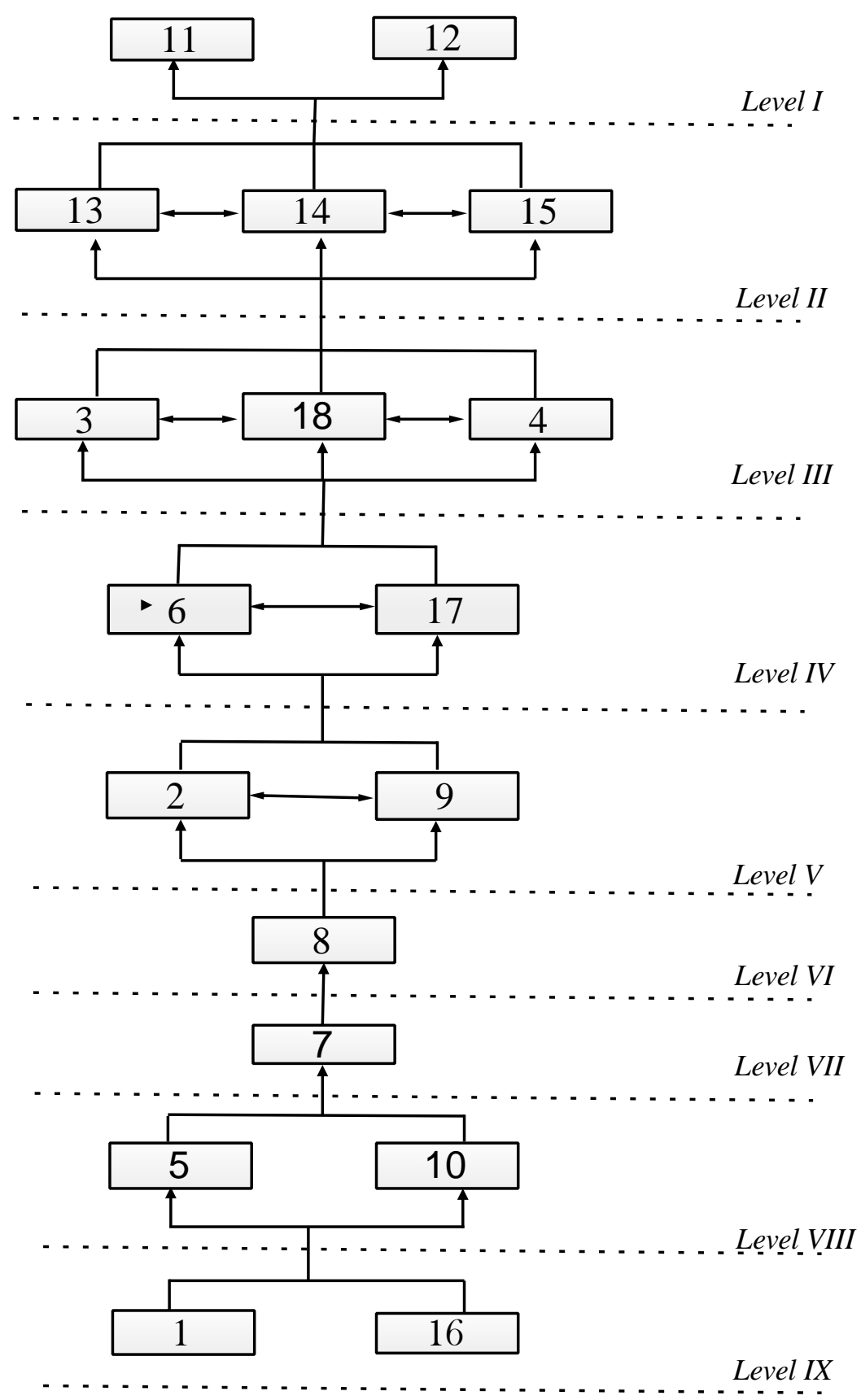

Figure 2: ISM Model

There are total nine levels where factor 11 and 12 occupy top level (Level I); 13, 14 and 15 (Level II); 3, 4 and 18 (Level III); 6 and 17 (Level IV); 2 and 9 (Level V); 8 (Level VI); 7 (Level VII); 5 and 10 (Level VIII) and 1 and 16 occupy bottom (Level IX). It can be observed that at levels 11, 12; 5, 10;1, 16 are not linked, but $13,14,15 ; 3,4,18 ; 6,17 ; 2,9$ are two way related.

MICMAC Analysis: It is a structural methodology that analyzes the underlying structure of factors (Godet, 1986). Objective of MICMAC is to identify key factors. This methodology has ability to supplement ISM by classifying the factors into four clusters namely independent, autonomous, linkage and dependent. 


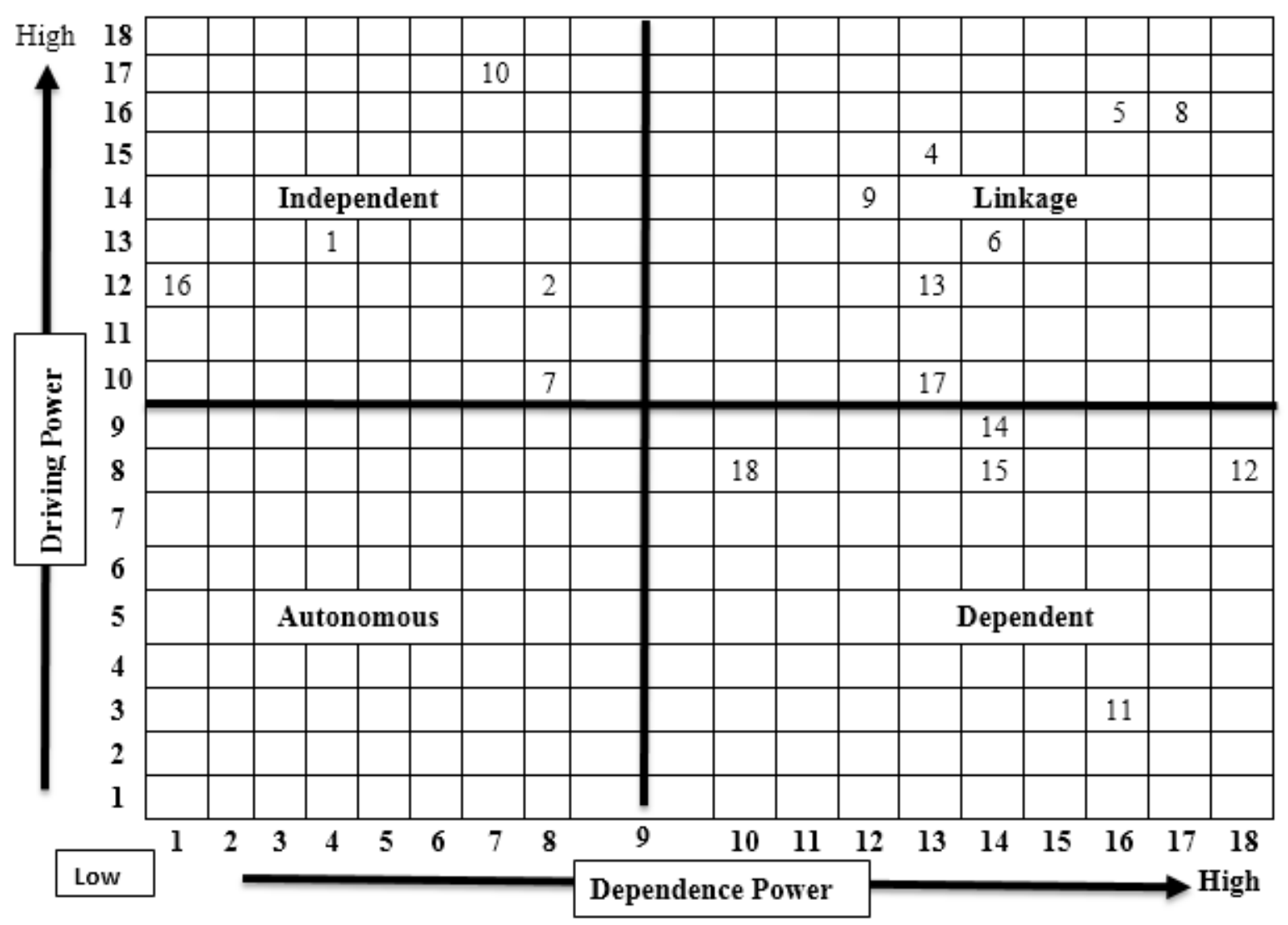

Figure 3: Driving-Dependence Diagram

Independent Custer: It contains factor having high driving power but low dependence power. Factors listed at 1, 2, 7, 10 and 16 are in independent cluster. These are key factors and high care is needed to handle them. Practitioners must therefore give priority to understanding these factors. Autonomous Cluster: This cluster contains the factors which have weak driving and weak dependence. They are separated from model have few but powerful links and don't have much impact on system. There is no such factor in this study in autonomous cluster. Non-existence of autonomous mean all factors play important role.

Dependent Cluster: It contains factors that have weak driving but strong dependence power. In this study factors listed at 11, 12, 14, 15 and 18 are dependent factors. Dependent factors resultantly depend on others and they need extra care.

Linkage Cluster: It contains factors that have strong driving and strong dependence power. In this study factors listed at 4, 5, 6, 8, 9, 13 and 17 are linking factors. They are considered as unbalanced because any action on them affects others and has feedback effect on themselves. 


\section{Results}

There are three parts of the study i.e. literature review, ISM and MICMAC. Results of literature review revealed that the total eighteen critical resemblances between two paradigms of banking. Results of ISM show that there is nine level structure underlying the phenomenon. Resemblance in rating system (11) and resemblance in public perception (12) occupy highest level therefore is less critical, whereas resemblance in settlement of cost of funds (1) and similar employee qualification (16) occupy bottom level and are the most critical. All remaining factors constitute middle part of the model having moderate low and moderate high severity depending on the level they occupy. Results of MICMAC show that 1, 2, 7, 10 and 16 are independent; 4, 5, 6, 8, 9, 13 and 17 are linking and 11, 12, 14, 15 and 18 are dependent.

\begin{tabular}{|c|c|c|c|c|c|c|c|}
\hline \multicolumn{2}{|c|}{ Result of Literature Review } & \multicolumn{4}{|c|}{ Results of MICMAC Analysis } & \multirow{2}{*}{$\begin{array}{c}\text { ISM Results } \\
\text { Level }\end{array}$} & \multirow{2}{*}{ Comments } \\
\hline No. & Barrier & Driving & Dependence & Effectiveness & Cluster & & \\
\hline 1 & $\begin{array}{l}\text { Resemblance in Settlement } \\
\text { of Cost of Funds }\end{array}$ & 13 & 4 & 9 & Independent & $I X$ & Key Factor \\
\hline 2 & $\begin{array}{l}\text { Resemblance in } \\
\text { Determination of Rates of } \\
\text { Profits }\end{array}$ & 12 & 8 & 4 & Independent & V & \\
\hline 3 & $\begin{array}{l}\text { Resemblance in Sanction } \\
\text { Procedures }\end{array}$ & 14 & 14 & 0 & Linkage & III & \\
\hline 4 & $\begin{array}{l}\text { Resemblance in Repayment } \\
\text { Annuities }\end{array}$ & 15 & 13 & 1 & Linkage & III & \\
\hline 5 & $\begin{array}{l}\text { Resemblance in } \\
\text { Documentation Processes }\end{array}$ & 16 & 16 & 0 & Linkage & VIII & \\
\hline 6 & $\begin{array}{l}\text { Resemblance in Working } \\
\text { Styles }\end{array}$ & 14 & 13 & 1 & Linkage & IV & \\
\hline 7 & $\begin{array}{l}\text { Resemblance in Interbank } \\
\text { Clearings }\end{array}$ & 10 & 8 & 2 & Independent & VII & \\
\hline 8 & $\begin{array}{l}\text { Resemblance in Borrowing } \\
\text { Patterns }\end{array}$ & 16 & 17 & -1 & Linkage & VI & \\
\hline 9 & $\begin{array}{l}\text { Resemblance in Accounting } \\
\text { Practices }\end{array}$ & 14 & 12 & 2 & Linkage & V & \\
\hline 10 & $\begin{array}{l}\text { Same Banking } \\
\text { Laws/Rules/Regulations }\end{array}$ & 17 & 7 & 10 & Independent & VIII & \\
\hline 11 & $\begin{array}{l}\text { Resemblance in Rating } \\
\text { System }\end{array}$ & 3 & 16 & -13 & Dependent & I & \\
\hline 12 & $\begin{array}{l}\text { Resemblance in Public } \\
\text { Perception }\end{array}$ & 8 & 18 & -10 & Dependent & I & \\
\hline 13 & $\begin{array}{l}\text { Resemblance in Client } \\
\text { Assessment Procedure }\end{array}$ & 12 & 13 & -1 & Linkage & II & \\
\hline 14 & $\begin{array}{l}\text { Resemblance in Banker's } \\
\text { Behavior }\end{array}$ & 9 & 14 & -5 & Dependent & II & \\
\hline 15 & $\begin{array}{l}\text { Resemblance in Recovery } \\
\text { Procedures }\end{array}$ & 8 & 14 & -6 & Dependent & II & \\
\hline 16 & $\begin{array}{l}\text { Similar Employee } \\
\text { Qualification }\end{array}$ & 12 & 1 & 11 & Independent & $I X$ & Key Factor \\
\hline 17 & $\begin{array}{l}\text { Resemblance in HRM } \\
\text { Practices }\end{array}$ & 10 & 13 & -2 & Linkage & IV & \\
\hline 18 & Similar Marketing Tactics & 8 & 10 & -2 & Dependent & III & \\
\hline
\end{tabular}

Table 15: Summary of Results

There is no autonomous factor. Summary of the results is presented as Table 15.

Resemblance in settlement of cost of funds (1) and similar employee qualification (16) are the key factors that create high degree of resemblance in Islamic and conventional banks.

\section{Discussion}

Main objective of the study is to identify, rank, exhume the relations and classify the critical resemblances between Islamic and conventional banking. The problem of resemblance of banking paradigms is concern of all stakeholders for different reasons vital to them. This research has identified the confusing factors and ranked them on the basis of their criticalness. It pointed out the key factors that are source of confusion and established the contextual relationships among them. It is an original attempt by the authors substantiated by real time survey data collected from experts on the issue. It is a seminal study that 
analyzed differently the phenomenon from traditional methods. This finding is consistent with the result of previous studies (Table 16).

Table 16: Comparison of Present Study with Prior Studies

\begin{tabular}{|c|c|c|c|c|c|c|}
\hline $\mathbf{S r}$ & Study & Focus & Country & Factors & Methodology & Result \\
\hline 1 & Current Study & Resemblances & Pakistan & Eighteen & ISM and MICMAC & $\begin{array}{l}\text { Resemblance in settlement } \\
\text { of cost of funds and } \\
\text { similar employee } \\
\text { qualification are key } \\
\text { factors. }\end{array}$ \\
\hline 2 & $\begin{array}{l}\text { Sukmana \& Ibrahim, } \\
2017\end{array}$ & Rates of return & Malaysia & $\begin{array}{l}1,3,6,9 \text { and } \\
12 \text { months } \\
\text { rates }\end{array}$ & $\begin{array}{l}\text { nonlinear } \\
\text { ARDL (NARDL) }\end{array}$ & $\begin{array}{l}\text { Rates of return do not } \\
\text { rigidly peg to each other. }\end{array}$ \\
\hline 3 & Lee \& Isa, 2017 & $\begin{array}{l}\text { Determinants of bank } \\
\text { margins }\end{array}$ & Malaysia & Fifteen & $\begin{array}{l}\text { Two-step } \\
\text { generalized method } \\
\text { of moments (GMM) } \\
\text { technique }\end{array}$ & $\begin{array}{l}\text { There are significant } \\
\text { similarities }\end{array}$ \\
\hline 4 & Khan et al., 2017 & Comparative performance & Pakistan & $\begin{array}{l}\text { Return to } \\
\text { Risk Ratio }\end{array}$ & $\begin{array}{l}\text { ARMA-GARCH-in- } \\
\text { Mean model }\end{array}$ & $\begin{array}{l}\text { No difference in the } \\
\text { performance. }\end{array}$ \\
\hline 5 & $\begin{array}{l}\text { Wan Ibrahim \& Ismail, } \\
2015\end{array}$ & $\begin{array}{l}\text { Similarities and } \\
\text { differences }\end{array}$ & Malaysia & Кок Кате & Literature review & $\begin{array}{l}\text { Similarity is prevalent in } \\
\text { supervisory roles. }\end{array}$ \\
\hline 6 & Al-Mamun et al., 2014 & Comparison & Malaysia & Seven & Financial Ratios & No major difference. \\
\hline
\end{tabular}

The authors found five different studies as listed above in Table 16 that are comparable with the study in hand. This comparison revealed that in general present studies is aligned with the contemporary literature but is different on the counts of factors under study, methodological approach, context of the study, respondents from which the data have been collected, scope of the study and results.

\section{Conclusion}

This research has identified the confusing factors and ranked them on the basis of their criticalness because resemblance between the two paradigms of banking is hot issue. The study pointed out the key factors that are source of confusion and established the contextual relationships among them. It is envisaged on literature review for identification of factors, employed ISM for ranking, prioritizing and modelling coupled with MICMAC for classification of the factors into independent, linkage, autonomous and dependent. Total eighteen factors were identified and sixteen experts were recruited from which the data was collected regarding the issue. Results of ISM show that there is nine level structure underlying the phenomenon. Resemblance in rating system (11) and resemblance in public perception (12) occupy highest level therefore is less critical, whereas resemblance in settlement of cost of funds (1) and similar employee qualification (16) occupy bottom level and are the most critical. All remaining factors constitute middle part of the model having moderate low and moderate high severity depending on the level they occupy. Results of MICMAC show that 1, 2, 7, 10 and 16 are independent; 4, 5, 6, 8, 9, 13, 17 are linking and $11,12,14,15$ and 18 are dependent. There is no autonomous factor. The study has valuable theoretical contribution by way of identification of factors, ISM model and driving-dependence diagram. Further it provides more supplementary information regarding the phenomena that gives greater understanding to stakeholders. The factors are generic and the results are fairly generalizable. ISM model and driving-dependence diagram offers practitioners and policy makers a framework for resolving practical issues. This study is helpful in building awareness in customers and society at large. It makes easy for researchers to develop further frameworks on the bases of classification. It is equally useful for regulators, management, employees, customers, researchers, religious scholars and society at large. It is an original attempt by the authors substantiated by real time survey data collected from experts on the issue. The study also has certain limitations. Firstly, ISM method only identifies but does not quantify the relationships therefore some other weighing method SEM, PCA, AHP, ANP, TOPSIS, GRA etc. may be used to quantify the relationships. Secondly, key factors have only been identified from limited number of published articles there might be some factors which might have been overlooked therefore future studies should be rather rigorous. Thirdly, the study was conducted in Pakistan since there are varying cultural, social, technological and political systems therefore future studies must be conducted in different countries. Fourthly, data has been collected from few stakeholders therefore it is recommended that extensive research should be conducted taking inputs from more stakeholders. 


\section{References}

Abdullah, M.R.T.L., \& Siraj, S. (2014). Interpretive Structural Modeling of MLearning Curriculum Implementation Model of English Language Communication Skills for Undergraduates. Turkish Online Journal of Educational Technology-TOJET, 13(1), 151-161.

Ahmad, N., \& Haron, S. (2002). Perceptions of Malaysian corporate customers towards Islamic banking products and services. International Journal of Islamic Financial Services, 3(4), 13-29.

Ahmad, A. U. F., \& Hassan, M. K. (2006). The adoption of the UK finance bill proposals on Islamic finance into Islamic banking in Australia. Review of Islamic Economics, 10(1), 41-57.

Ahmad, A., Saif, I., \& Safwan, N. (2010). An empirical investigation of Islamic banking in Pakistan based on perception of service quality. African journal of business management, 4(6), 1185-1193.

Ahmed, E., Rahman, Z., \& Ahmed, R. I. (2006). Comparative analysis of loan recovery among nationalized, private and Islamic commercial banks of Bangladesh. BRAC University Journal, 8(1), 35-52.

Ahmed, S.U., Rahman, A., Ahmed, S., \& Ullah, G.M. (2014). Pricing linkage between Islamic banking and conventional banking: The case of Bangladesh. International Journal of Finance \& Banking Studies (IJFBS), 3(4), 84-97.

Al-Mamun, A., Yasser, Q.R., Entebang, H., Rahman, M., Nathan, T.M., \& Abu Mansor, S. (2014). Comparison between Islamic and Conventional Banking: Evidence from Malaysia. International Journal of Excellence in Islamic Banking and Finance, 182(1464), 1-14.

Amin, M., Yahya, Z., Ismayatim, W.F.A., Nasharuddin, S.Z., \& Kassim, E. (2013). Service quality dimension and customer satisfaction: An empirical study in the Malaysian hotel industry. Services Marketing Quarterly, 34(2), 115-125.

Ariff, M., \& Lewis, M.K., 2014. Similarities and differences in Islamic and conventional banking. In: Lewis, M.K., Ariff, M., Mohamad, S. (Eds.), Risk and Regulation of Islamic Banking. Edward Elgar, Cheltenham, UK \& Northampton, MA, USA, pp. 55-70.

Asif, M., Ahmed, U., Zahid, M., \& Khan, A. (2017). Motives Behind the Transfer of a Bank from Conventional Banking to Islamic Banking in Pakistan. Journal of Business and Tourism, 3(2), 225 234.

Attri, R., Dev, N., \& Sharma, V. (2013). Interpretive structural modelling (ISM) approach: an overview. Research Journal of Management Sciences, 2319, 1171.

Azevedo, S., Carvalho, H., \& Cruz-Machado, V. (2013). Using interpretive structural modelling to identify and rank performance measures: an application in the automotive supply chain. Baltic Journal of Management, 8(2), 208-230.

Azmat, S., Skully, M., \& Brown, K. (2017). The (little) difference that makes all the difference between Islamic and conventional bonds. Pacific-Basin Finance Journal, 42, 46-59.

Badruddin, A. (2015). Islamic Banking and Finance in India: A Kosher or Myth. International Journal of Management, Innovation and Entrepreneurial Research, 1(1), 1-6.

Bashir, A., Sajid, M.R., \& Iqbal, S. (2011). Barriers to Islamic Banking Growth-Bank Employees' Perspective from Pakistan. Asian Journal of Finance \& Accounting, 3(1), 1.

Beck, T., Demirgüç-Kunt, A., \& Pería, M.S.M. (2011). Bank financing for SMEs: Evidence across countries and bank ownership types. Journal of Financial Services Research, 39(1-2), 35-54.

Bennett, M. S., \& Iqbal, Z. (2013). How socially responsible investing can help bridge the gap between Islamic and conventional financial markets. International Journal of Islamic and Middle Eastern Finance and Management, 6(3), 211-225.

Bhosale, V.A., \& Kant, R. (2016). An integrated ISM fuzzy MICMAC approach for modelling the supply chain knowledge flow enablers. International Journal of Production Research, 54(24), 7374-7399.

Bowra, Z.A., Sharif, B., Saeed, A., \& Niazi, M.K. (2012). Impact of human resource practices on employee perceived performance in banking sector of Pakistan. African Journal of Business Management, 6(1), 323-332.

Cai, Y., \& Xia, C. (2018). Interpretive Structural Analysis of Interrelationships among the Elements of Characteristic Agriculture Development in Chinese Rural Poverty Alleviation. Sustainability, 10(3), 786. 
Clayton, M. J. (1997). Delphi: a technique to harness expert opinion for critical decision-making tasks in education. Educational Psychology, 17(4), 373-386.

Dhochak, M., \& Sharma, A.K. (2016). Using interpretive structural modeling in venture capitalists' decision-making process. Decision, 43(1), 53-65.

Dhochak, M., \& Sharma, A.K. (2016). Using interpretive structural modeling in venture capitalists' decision-making process. Decision, 43(1), 53-65.

Godet, M. (1986). Introduction to la prospective: seven key ideas and one scenario method. Futures, 18(2), 134-157.

Hadriche, M. (2015). Banks performance determinants: Comparative analysis between conventional and Islamic banks from GCC countries. International Journal of Economics and Finance, 7(9), 169177.

Hanif, M. (2014). Differences and similarities in Islamic and conventional banking. International Journal of Business and Social Sciences, 2(2), 166-175.

Hanif, M., Tariq, M., \& Tahir, A. (2012). Comparative performance study of conventional and Islamic banking in Pakistan. International Research Journal of Finance \& Economics, (83), 62-72.

Haron, S., \& Ahmad, N. (2000). The effects of conventional interest rates and rate of profit on funds deposited with Islamic banking system in Malaysia. International Journal of Islamic Financial Services, 1(4), 1-7.

Ibrahim, W.H.W, \& Ismail, A.G. (2015). Conventional bank and Islamic banking as institutions: Similarities and differences. Humanomics, 31(3), 272-298.

Ismath Bacha, O. (2008). The Islamic inter bank money market and a dual banking system: the Malaysian experience. International Journal of Islamic and Middle Eastern Finance and Management, 1(3), 210-226.

Jawadi, F., Cheffou, A.I., \& Jawadi, N. (2016). Do Islamic and conventional banks really differ? A panel data statistical analysis. Open Economies Review, 27(2), 293-302.

Karim, R.A.A. (2001). International accounting harmonization, banking regulation, and Islamic banks. The International Journal of Accounting, 36(2), 169-193.

Karim, M.A., Hassan, M.K., Hassan, T., \& Mohamad, S. (2014). Capital adequacy and lending and deposit behaviors of conventional and Islamic banks. Pacific-Basin Finance Journal, 28, 58-75.

Khan, F. (2010). How 'Islamic'is Islamic banking? Journal of Economic Behavior \& Organization, 76(3), 805-820.

Khan, S.A., Khaleequzzaman, M., Ishfaq, M., \& Khan, S. Z. (2017). Comparative Risk and Return Analysis of Islamic and Conventional Financial Institutions in Pakistan. International Journal of Islamic Economics and Finance Studies, 3(1), 109-152.

Khan, S., \& Khan, M.S.A. (2013). Conceptualized Model of Green It Purchasing Enablers-An Application of Delphi Technique and Interpretive Structural Modeling. Business Sciences International Research Journal, 1(1), 24-37.

Khan, M.S., \& Mirakhor, A. (1994). Monetary management in an Islamic economy. Journal of King Abdulaziz University: Islamic Economics, 6(1), 3-12.

Kumar, S., Luthra, S., \& Haleem, A. (2013). Customer involvement in greening the supply chain: an interpretive structural modeling methodology.Journal of Industrial Engineering International, 9(1), 6.

Lee, S.P., \& Isa, M. (2017). Determinants of bank margins in a dual banking system. Managerial Finance, 43(6), 630-645.

Li, G., Huang, D., Sun, C., \& Li, Y. (2019). Developing interpretive structural modeling based on factor analysis for the water-energy-food nexus conundrum. Science of The Total Environment, 651, 309322.

Li, M., \& Yang, J. (2014). Analysis of interrelationships between critical waste factors in office building retrofit projects using interpretive structural modelling. International Journal of Construction Management, 14(1), 15-27.

Lohaus, D., \& Habermann, W. (2019). Presenteeism: A review and research directions. Human Resource Management Review, 29(1), 43-58. 
Omercic, J. (2016). Heaven's Bankers: Inside the hidden world of Islamic finance. International Journal of Economics, Management and Accounting, 24(2), 261-264.

Ranjbar, M.S., Azami, A., \& Afraze, A. (2012). Analysis of interaction among effective factors on corporate entrepreneurship. Asia Pacific Journal of Innovation and Entrepreneurship, 6, 9-31.

Rhanoui, S., \& Belkhoutout, K. (2018). Operational Risk in Both Conventional and Islamic Banking Perceptions: Differences and Similarities. European Scientific Journal, 14(13), 110-120.

Rizvi, S.A.R., \& Arshad, S. (2014). An empirical study of Islamic equity as a better alternative during crisis using multivariate GARCH DCC. Islamic Economic Studies, 130(1155), 1-27.

Saeed, M., \& Baig, A. K. (2013). Islamic Shari'ah-compliant marketing. International Journal of Social Entrepreneurship and Innovation, 2(2), 166-178.

Saidu, O. S. (2014). How important is it to have a special law governing Islamic banking? Online Journal of Research in Islamic Studies, 1(3), 1-7.

Salman, A., \& Nawaz, H. (2018). Islamic financial system and conventional banking: A comparison. Arab Economic and Business Journal, 13(2), 155-167.

Samad, A. (2004). Performance of Interest-free Islamic banks vis-à-vis Interest-based Conventional Banks of Bahrain. International Journal of Economics, Management and Accounting, 12(2), 1-15.

Song, L., Li, Q., List, G., Deng, Y., \& Lu, P. (2017). Using an AHP-ISM based method to study the vulnerability factors of urban rail transit system. Sustainability, 9(6), 1065.

Sukmana, R., \& Ibrahim, M. H. (2017). How Islamic are Islamic banks? A non-linear assessment of Islamic rate-conventional rate relations. Economic Modelling, 64, 443-448.

Sushil, A. (2017). Modified ISM/TISM Process with Simultaneous Transitivity Checks for Reduced Direct Pair Comparisons. Global Journal of Flexible Systems Management, 18(4), 331-351.

Sushil, S. (2012). Interpreting the interpretive structural model. Global Journal of Flexible Systems Management, 13(2), 87-106.

Thakkar, J., Kanda, A., \& Deshmukh, S.G. (2008). Interpretive structural modeling (ISM) of IT- enablers for Indian manufacturing SMEs. Information Management \& Computer Security, 16(2), 113-136.

Valmohammadi, C., \& Dashti, S. (2016). Using interpretive structural modeling and fuzzy analytical process to identify and prioritize the interactive barriers of e-commerce implementation. Information \& Management, 53(2), 157-168.

Warfield, J.N. (1973). Binary matrices in system modeling. IEEE Transactions on Systems, Man, and Cybernetics, (5), 441-449.

Warfield, J.N. (1974). Toward interpretation of complex structural models. IEEE Transactions on Systems, Man, and Cybernetics, (5), 405-417.

Xiao, L. (2018). Analyzing consumer online group buying motivations: An interpretive structural modeling approach. Telematics and Informatics, 35(4), 629-642.

Zainol, Z., Shaari, R., \& Muhamad Ali, H. (2009). A comparative analysis on bankers' perceptions on Islamic banking. International Journal of Business and management, 3(4), 157-168.

Zarrouk, H., Ben Jedidia, K., \& Moualhi, M. (2016). Is Islamic bank profitability driven by same forces as conventional banks? International Journal of Islamic and Middle Eastern Finance and Management, 9(1), 46-66.

Zeitun, R., \& Benjelloun, H. (2012). The Efficiency of Banks and Financial Crisis in a Developing Economy: The Case of Jordan. International Review of Accounting, Banking \& Finance, 4(2), 2860.

Zhang, Y.J., \& Wei, Y.M. (2010). An overview of current research on EU ETS: Evidence from its operating mechanism and economic effect. Applied Energy, 87(6), 1804-1814. 